\title{
Preprocedural mouth rinses can reduce bacterial contamination in aerosols during periodontal prophylaxis
}

\section{Erfan Shamsoddin ${ }^{1}$}

\section{A commentary on:}

\section{Mohd-Said S, Mohd-Dom T N, Suhaimi N, Rani H, McGrath C.}

Effectiveness of Preprocedural Mouth Rinses in Reducing Aerosol

Contamination During Periodontal Prophylaxis: A Systematic Review.

Front Med (Lausanne) 2021; DOI: 10.3389/fmed.2021.600769.

\begin{abstract}
Aim This study evaluates the effectiveness of preprocedural mouth rinsing when performing non-surgical periodontal prophylaxis to reduce bacterial aerosol contamination.
\end{abstract}

Data sources The authors used the population, intervention, control and outcomes (PICO) question format to perform a systematic online search in Scopus, PubMed, Cochrane Library and Web of Science. All the databases were explored with no time limit until April 2020.

Study selection Randomised clinical trials were included in this systematic review. No inclusion or exclusion criteria are mentioned in this study and it is limited to bacterial contamination studies.

Data extraction and synthesis The authors extracted the following information from the included studies: authors; article publication year; study design; sampling size and allocation of test and control groups; intervention details; type and description of periodontal prophylaxis procedures; and primary outcomes. They focused on statistically significant findings and the reduction of bacterial aerosol contamination between groups was measured by colony forming units (CFU) using means and percentages. The primary outcome was that bacterial count expressed as CFU on blood agar plates. If permissible, they calculated the mean CFU reduction in the included studies and reported them. The authors also assessed the studies' risk of bias using the revised Cochrane risk of bias tool for randomised trials.

Results In total, 30 randomised controlled trials were included, 21 of which assessed the preprocedural rinsing and the remaining nine focused on other interventions. The bacterial incubation protocol differed among studies. The data collection sites differed among the studies in terms of the number of samples obtained, position, direction and distance from the subjects' mouths. There were no marked differences in the CFU reduction regarding the periodontal prophylaxis devices used and the location of aerosol sampling collection from these studies. Nearly half of the studies $(52.4 \%, 11 / 21)$ collected the sample at or near the operator and dental assistant. Chlorhexidine $(\mathrm{CHX})$ rinse was mostly tested $(80.9 \%, 17 / 21)$ with various concentrations and volumes. Among studies comparing $\mathrm{CHX}$ with other agents $(71.4 \%, 15 / 21), \mathrm{CHX}$ was more effective for the better part of studies, with more than half of the studies (7/15) reporting over a $70 \%$ reduction in $\mathrm{CFU}$ when using $\mathrm{CHX}$ preprocedural mouth rinse.

\section{GRADE rating}

Practice point

- The use of preprocedural mouth rinses, especially chlorhexidine, can lower bacterial aerosol contamination during non-surgical periodontal prophylaxis.
Conclusions Evidence corroborates the effectiveness of preprocedural mouth rinses, especially $\mathrm{CHX}$, in reducing the bacterial contamination of aerosols in periodontal, non-surgical prophylaxis compared with mouth rinsing with water, or with no rinsing. Nevertheless, the low-quality of evidence and the high heterogeneity among the included studies warn that one should be cautious in generalising the findings of this study to other settings and contaminating agents, like viruses. The findings of this study emphasise the need for more studies on the efficacy and effectiveness of preprocedural rinses in reducing the viral load in aerosols (and droplets) during periodontal prophylaxis procedures.

\section{Commentary}

This systematic review assessed clinical evidence about the effectiveness of preprocedural mouth rinses in reducing bacterial aerosol contamination during non-surgical, periodontal prophylaxis. The authors used the population, intervention, control and outcomes (PICO) format to structure the study question and conducted an online search in four databases: Scopus; Medline via PubMed; Cochrane Library; and Web of Science. The authors claimed to have abided by the Preferred Reporting Items for Systematic Reviews and Meta-Analyses (PRISMA) statement, though they failed to do so and did not provide a comprehensive, structured, unbiased piece of evidence at the end.

Out of 731 preliminary results from the four databases, 56 studies were determined as eligible for qualitative data synthesis. The authors excluded 26 non-randomised controlled trials, limiting the studies to 30 randomised controlled trials for inclusion. From the included studies, 21 focused on preprocedural mouth rinsing and 9 evaluated other interventions during nonsurgical periodontal prophylaxis. No eligibility criteria were mentioned in the study, leading to its deviation from the PRISMA statement. However, the authors reported the factors considered for their research question based on the PICO format. The study population included periodontitis patients receiving interventions to reduce bacterial aerosol contaminations during non-surgical prophylaxis, including dental scaling and tooth polishing, root planing or debridement and air polishing using powered instruments with/without the use of adjunctive antimicrobials. In addition to descriptive data, the authors also extracted the statistically significant findings and reduction of bacterial aerosol 
contamination between groups, measured by colony forming units (CFU), using means and percentage as the primary outcome. They measured the mean percentage of reduction in CFU by subtracting the total amount of CFU after prophylaxis from the baseline mean of CFU and dividing it by the baseline mean of CFU. If the baseline data was not available, they subtracted the mean CFU for the intervention group from the mean CFU for the control group and divided the resultant by the mean CFU for the control group. In both cases, the mean CFU reduction was reported in percent.

The incubation protocol differed among the studies assessing the bacterial aerosol contamination (20 of 21 studies). Additionally, the sample collection sites varied among studies regarding the number of samples obtained, position, direction and distance from the subjects' mouths. In most cases, chlorhexidine (CHX) rinse was tested $(80.9 \%, 17 / 21)$ with various concentrations and volumes. Among studies comparing CHX with other agents (71.4\%, 15/21), the effectiveness of $\mathrm{CHX}$ over other agents was apparent, with 7 studies out of 15 reporting over a $70 \%$ reduction in CFU. The protocol for preprocedural rinsing varied - participants were instructed to rinse between 30 seconds and 2 minutes, the amount of mouth rinse ranged between $10-20 \mathrm{ml}$ and participants waited between 2-40 minutes before receiving periodontal prophylaxis. Some studies tested additional interventions to examine their impact on reducing bacterial load as well. For instance, highvolume evacuation and ozone irrigation were performed as auxiliary interventions.

The risk of bias was assessed in all the included studies using the revised Cochrane risk of bias tool for randomised trials. The authors scored the risk of bias (ROB) as either low ROB, some concerns, or high ROB. The authors stated that $90.5 \%$ of studies had an unclear ROB regarding the randomisation process. They scored 4 studies with high ROB (19\%), 16 with some concerns (76\%) and 1 with low ROB (5\%).

The findings of this paper support the effectiveness (and efficacy) of preprocedural mouth rinses in lowering the bacterial aerosol contamination during periodontal prophylaxis. The authors only mentioned the 'effectiveness' of mouth rinses, though nine of their included studies (42.8\%) assessed the 'efficacy' (and not effectiveness) of preprocedural mouth rinses. This is quite a crucial distinction as many clinicians consider the 'efficacy' of mouth rinses to be more clinically significant than the 'effectiveness' of such an intervention. This study also tries to modify and 'enhance' the PRISMA statement by adding arbitrary and newly-formed jargon like 'potentially effective' and 'effective' studies, striving to further differentiate among the eligible studies, while failing to explicitly state the inclusion and exclusion criteria during the screening process. These practices should utterly be avoided and clinical researchers are encouraged to follow the latest protocols and frameworks to produce structured and comprehensive manuscripts that do not cause further confusion for the readers. Another downside of this study is that their discussion considerably strays off the main topic in an effort to 'glue' the results to viral aerosol contaminations. They aimed to highlight the importance of reducing aerosol contamination during the COVID-19 pandemic, which, unfortunately, wholly shadowed over their results in the discussion, preventing it from becoming a rich and relevant section in their paper. The discussion could have emphasised the need for more assessments on viral agents, rather than making the primary outcome of the systematic review a surrogate outcome for viral aerosol contamination (which was not intended in their methodology).

The literature has widely heeded the issue of viral aerosol contamination and managing aerosol-generating procedures in dentistry and emphasised the uncertainty around this issue. ${ }^{1,2}$ There are also doubts about the efficacy of antiseptic mouth rinses (like CHX) without adjunctive alcohol against viruses. ${ }^{3}$ As stated by this paper, further studies to assess the efficacy and effectiveness of preprocedural mouth rinses on lowering the viral load in aerosols (and droplets) generated during periodontal prophylaxis are necessary. Be that as it may, suggesting controversial oral health policies, like prioritising the treatment needs of dental patients during the COVID-19 pandemic and adding the use of mouth rinses before periodontal prophylaxis as a mandatory, standard, operating procedure in current dental practice, is highly advised against. These types of inference from limited evidence, which in this case opposes some guidelines as well, ${ }^{4,5}$ can do more damage than good to dental sciences and dentistry in general. Evidencebased dentistry needs more systematic, balanced and evidencebased claims during these uncertain times more than ever.

\section{Author affiliation}

${ }^{1}$ National Institute for Medical Research Development, Tehran, Iran.

Correspondence to: Erfan Shamsoddin

\section{References}

1. Kumbargere Nagraj S, Eachempati P, Paisi M, Nasser M, Sivaramakrishnan G, Verbeek J H. Interventions to reduce contaminated aerosols produced during dental procedures for preventing infectious diseases. Cochrane Database Syst Rev 2020; DOI: 10.1002/14651858.CD013686.pub2.

2. Innes N, Johnson I G, Al-Yaseen W et al. A systematic review of droplet and aerosol generation in dentistry. J Dent 2021; 105: DOI: 10.1016/j.jdent.2020.103556.

3. Gurzawska-Comis K, Becker K, Brunello G, Gurzawska A, Schwarz F. Recommendations for Dental Care during COVID-19 Pandemic. / Clin Med 2020; 9: 1833.

4. World Health Organisation. Considerations for the provision of essential oral health services in the context of COVID-19: interim guidance, 3 August 2020. 2020. Available at https://apps.who.int/iris/handle/10665/333625 (accessed November 2021).

5. Centers for Disease Control and Prevention. Guidance for Dental Settings. 2020 Available at https://www.cdc.gov/coronavirus/2019-ncov/hcp/dental-settings.html (accessed November 2021).

Evidence-Based Dentistry (2021) 22, 139-139.

https://doi.org/ 10.1038/s41432-021-0220-0 